\title{
The Relationship between Recurrent Spine Surgery and Employment Status
}

\author{
Mohammad Sami Walid, Nadezhda Zaytseva, Moataz Abbara, Azhar Abdullah \\ Department of Medical Education and Research, Medical Center of Central Georgia, Macon, USA \\ Email:mswalid@yahoo.com
}

Received November 11, 2011; revised December 10, 2011; accepted December 19, 2011

\begin{abstract}
Background: What is the quantitative relationship between repeated surgical intervention and unemployment in the spine surgery population? And, does the literature pay sufficient attention to this important aspect of spine surgery outcome? Methods: This was a retrospective review of 905 patients of working age undergoing one of three types of spine surgery. The index surgery at the time of the study was either on the cervical or lumbar spine. We retrospectively collected data on patients' employment status and history of prior spine surgery. In this study, history of prior spine surgery was coded using two nominal (categorical) variables: The number of previous spine surgeries $(0,1,2, \geq 3)$ and the variability of location of previous spine surgeries (cervical, lumbar). We also looked into scientific publications related to spine surgery and probed "employment" and "reoperation" awareness in randomized controlled trials (RCTs). In addition, we queried some common factors that are known to play an important role in exacerbating the unemployment problem, such as opioid (ab)use and depression. Results: The unemployment rate was $19.4 \%$ among males and $34.8 \%$ among females. Unemployment rate correlated with the number of previous spine surgery $(r=0.077, p=0.020)$, opioid use $(r=0.080, p=0.017)$ and being on antidepressants $(r=0.119, p=0.000)$. The unemployment rate was statistically different $(\chi 2=10.656, p=0.014)$ among patients with different numbers of previous spine surgeries: $25.6 \%$ of de novo patients versus $28.1 \%$ of patients with one previous spine surgery, $32.7 \%$ of patients with two previous spine surgeries and $48.7 \%$ of patients with three or more previous spine surgeries. Females had significantly higher unemployment rate if they had three or more previous spine surgeries in their past $\left(31.4 \%, 34.4 \%, 46.2 \%\right.$ versus $83.3 \%, \chi^{2}=$ $21.841, \mathrm{p}=0.000$ ). Unemployment rate was as high as $90 \%$ in female patients with $\geq 3$ surgeries on different regions of the spine and receiving antidepressants. Among randomized controlled trials addressing spine surgery in humans and published in English, 10.4\% of reported studies mentioned opioids in their text, 4.1\% (23/566) mentioned reoperation, $1.4 \%(8 / 566)$ mentioned employment, and none mentioned antidepressants. Conclusion: Females' employment status is more sensitive to repeated surgical intervention, regardless of the part of the spine being targeted, reaching close to ten times the general population's unemployment rate $(83.3 \%$ versus $8 \%)$. On the other hand, a small percentage of randomized controlled trials pertaining to spine surgery deals with employment issues and reoperation rate in their outcome analysis.
\end{abstract}

Keywords: Unemployment; Reoperation; Females; Opioids; Antidepressants; Randomized Controlled Trials

\section{Introduction}

Spine surgery is last resort treatment for different back problems and is relatively more accessible in the United States than in other countries [1]. In the United States, the yearly incidence of spine surgery is estimated at $0.2 \%$ of the population [2]. Also, in the United States, the unemployment rate for adult men is $8.8 \%$ and for adult women $8.0 \%$ according to most recent data (October 2011) [3]. It is known that spine disorders and ensuing spine surgery is a common reason for disability and unemployment $[4,5]$. So what is the quantitative relationship between repeated surgical intervention and unemployment in the spine surgery population? Moreover, does the literature pay sufficient attention to this important aspect of spine surgery outcome?

In a previous study [6], we retrospectively reviewed the charts of 629 patients of working age who underwent spine surgery. We studied the relationship between their employment status, history of depression, and history of prior spine surgery as a dichotomal variable, patients with no previous spine surgery and patients with previous spine surgery. We found that unemployment and depression were strongly associated with a history of prior spine surgery in the female cohort of our study population.

In a later study [7], we retrospectively analyzed and 
prospectively followed 1153 spine surgery patients with no age restriction and demonstrated that de novo spine surgery is associated with an increased incidence of additional spine surgery at the same or distant spine regions.

The results of our preliminary studies led us to reflect on a possible correlation between the number and location of prior spine surgeries and employment status.

\section{Materials \& Methods}

This was a retrospective review of 905 patients of working age undergoing one of three types of spine surgery. The index surgery at the time of the study was either on the cervical or lumbar spine. We retrospectively collected data on patients' employment status and history of prior spine surgery. In this study, history of prior spine surgery was coded using two nominal (categorical) variables (Table 1):

a) Number of previous spine surgeries $(0,1,2, \geq 3)$.

b) Variability of location of previous spine surgeries (cervical, lumbar):

- Same region: The location of previous and index spine surgeries is the same.

- Different regions: The location of previous spine surgeries was different either between two previous spine surgeries or between previous and index spine surgery.

In statistical analysis, patients undergoing their first spine surgery were included in the group of patients who had no prior spine surgeries and coded as same spine region (neck or lumbar). The $\chi 2$ and Fisher exact tests were used to determine significant differences between the rates of unemployment between patients with diff- erent numbers of prior spine surgery. In addition, Spearman

Table 1. Characteristics of the study cohort.

\begin{tabular}{|c|c|}
\hline Variables & Details \\
\hline Age & Between 25 and 65 years, median 51 years \\
\hline Gender & $46.9 \%$ males, $53.1 \%$ females \\
\hline Date of Surgery & Operated between 2005 and 2008 \\
\hline Type of Surgery & $\begin{array}{l}17 \% \text { lumbar microdiscectomy (LMD) patients } \\
64.8 \% \text { anterior cervical decompression \& fusion } \\
\text { (ACDF) patients } \\
18.2 \% \text { lumbar decompression \& fusion (LDF) } \\
\text { patients }\end{array}$ \\
\hline $\begin{array}{l}\text { Number of } \\
\text { previous spine } \\
\text { surgeries }\end{array}$ & $\begin{array}{l}\text { No previous spine surgeries (de novo patients) } \\
69.2 \% \\
1 \text { previous spine surgery } 20.4 \% \text { (now undergoing } \\
\text { index surgery) } \\
2 \text { previous spine surgeries } 6.1 \% \text { (now undergoing } \\
\text { index surgery) } \\
\geq 3 \text { previous spine surgeries } 4.3 \% \text { (now undergoing } \\
\text { index surgery) }\end{array}$ \\
\hline
\end{tabular}

correlation test was applied between unemployment rate and number of previous spine surgeries. Statistical analysis and graphing was accomplished using Excel (2003; Microsoft, Redmond, WA) and SPSS Statistics (version 16; IBM, Somers, NY).

In the context of the topic of this paper, we looked into scientific publications related to spine surgery and probed "employment" and "reoperation" awareness in randomized controlled trials (RCTs), which constitute level one evidence in medicolegal discussions. In addition, we queried some common factors that are known to play an important role in exacerbating the unemployment problem, such as opioid (ab)use and depression. The PubMed biomedical search engine was queried using the criteria "English", "Human" and the following medical subject headings (MeSH) and subheadings:

- "Spine/Surgery" [MeSH Terms],

- "Employment" [MeSH Terms],

- “Reoperation" [MeSH Terms],

- “Analgesics, opioid” [MeSH Terms],

- "Antidepressive agents" [MeSH Terms].

\section{Results}

The unemployment rate in this working age patient cohort was $27.6 \%$ where $19.4 \%$ of males and $34.8 \%$ of females were unemployed. Unmployment rate correlated with the number of previous spine surgery $(\mathrm{r}=0.077, \mathrm{p}=$ $0.020)$, opioid use $(\mathrm{r}=0.080, \mathrm{p}=0.017)$ and being on antidepressants $(\mathrm{r}=0.119, \mathrm{P}=0.000)$.

The unemployment rate was statistically different $(\chi 2$ $=10.656, \mathrm{P}=0.014$ ) among patients with different numbers of previous spine surgeries (Figure 1). Unemployment rate nearly doubles when the patients under-

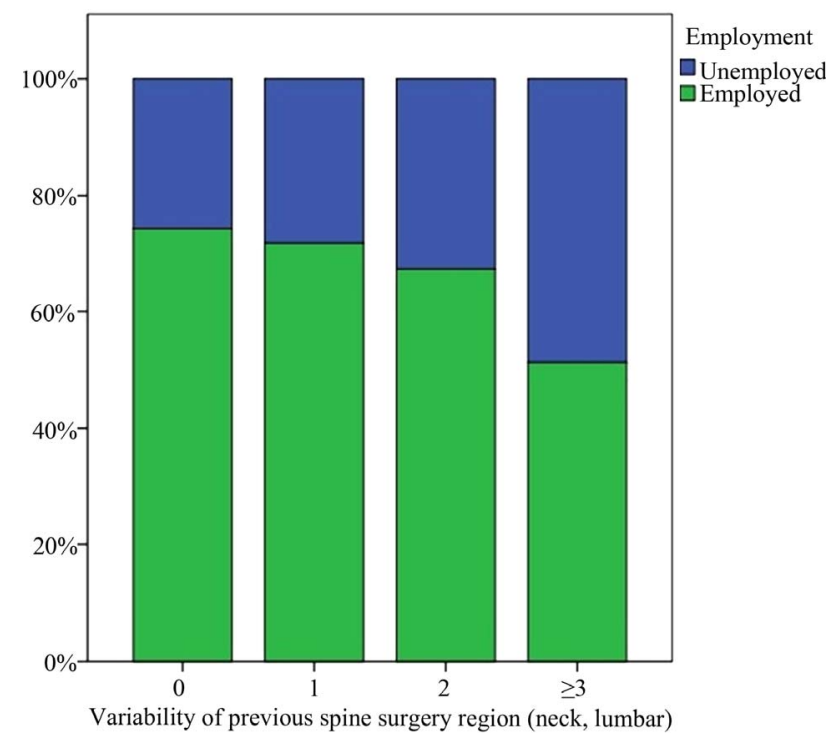

Figure 1. Increasing unemployment rate with increasing number of interventions on the spine of working age patients. 
goes three or more spine surgeries, $25.6 \%$ of de novo patients versus $28.1 \%$ of patients with one previous spine surgery, $32.7 \%$ of patients with two previous spine surgeries and $48.7 \%$ of patients with three or more previous spine surgeries.

Breaking down by gender (Figure 2), men had no statistically different unemployment rates $(\chi 2=0.130, \mathrm{P}=$ 0.988) whether they were de novo or recurrent patients with one, two, three or more previous spine surgeries (19\%, 20.5\%, 20.7\% and 19\%, respectively). Females, on the other hands, had significantly higher unemployment rate if they had three or more previous spine surgeries in their past $(31.4 \%, 34.4 \%, 46.2 \%$ versus $83.3 \%$, $\left.\chi^{2}=21.841, \mathrm{P}=0.000\right)$.

Adding the location of previous spine surgeries (Figure 3 ), the highest unemployment rate was among females with three or more previous spine surgeries on more than one region of the spine $(84.6 \%)$. The variability of previous spine surgeries location did not make a statistically significant difference regarding unemployment rate within each recurrent patient subgroup $(0,1,2, \geq 3)$.

Studying the impact of opioid and antidepressant use in the female cohort only, unemployment rate was as high as $7 / 8(87.5 \%)$ in opioid-medicated female patients with $\geq 3$ surgeries on different regions of the spine and as high as $9 / 10(90 \%)$ in female patients with $\geq 3$ surgeries on different regions of the spine who were on antidepressants (Figure 4).

Among randomized controlled trials addressing spine surgery in humans and published in English, 10.4\% of reported studies mentioned opioids in their text, $4.1 \%$ (23/566) mentioned reoperation, 1.4\% (8/566) mentioned employment, and none mentioned antidepressants.

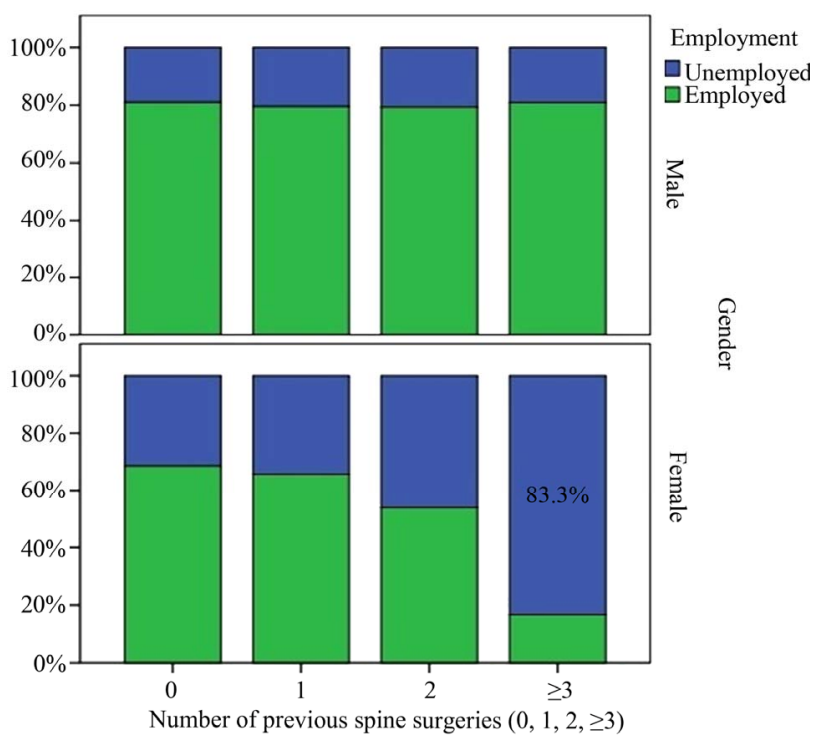

Figure 2. Increasing unemployment rate with increasing number of interventions on the spine of male and female patients of working age.

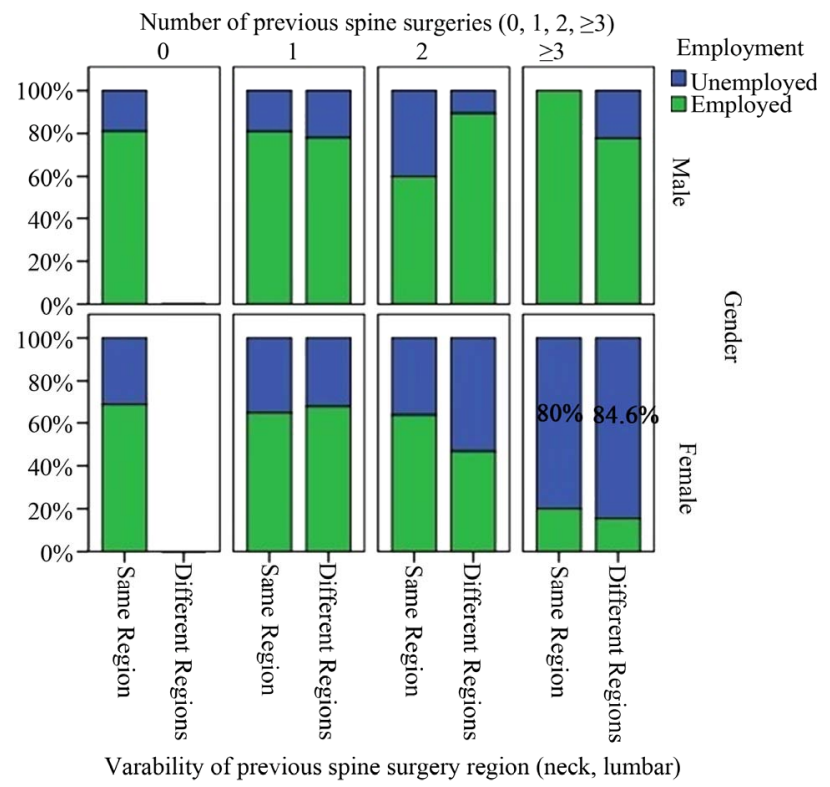

Figure 3. Increasing unemployment rate with increasing number of spine interventions and variability of intervention location in males and females of working age.

\section{Discussion}

Back pain may occur at all ages as a result of an occupational or sport injury, motor vehicle accident, or because of the wear and tear effect of time leading to degenerative spine disease in older patients. Back pain can be extremely debilitating and prevent an individual from returning to their current job. Many patients ultimately resort to surgery, after they have exhausted all non-surgical options. Surgical treatment usually alleviates pain, but in a small percentage, the opposite effect may take place. Patients become therefore eligible for disability benefits. The most common cause $(30.1 \%)$ of existing disability claims in 2010 was diseases of the musculoskeletal system and connective tissue [8]. The diagnosis of failed back syndrome was coined to describe these chronically ill patients who did not profit from a previous surgical intervention on their spine.

Our data show that females' employment status is more sensitive to repeated surgical intervention, regardless of the part of the spine being targeted, reaching close to ten times the general population's unemployment rate (83.3\% versus $8 \%$ ), and higher among those on opioid and antidepressant medications. On the other hand, a small percentage of randomized controlled trials pertaining to spine surgery dealt with employment issues in their design (1.4\%). A higher but still small percentage $(4.1 \%)$ of high grade spine surgery literature included reoperation rate in their outcome analysis. More opioid awareness $(10.4 \%)$ in randomized controlled trials was evident compared with antidepressants $(0 \%)$. Recent research shows important differences in the demographic 

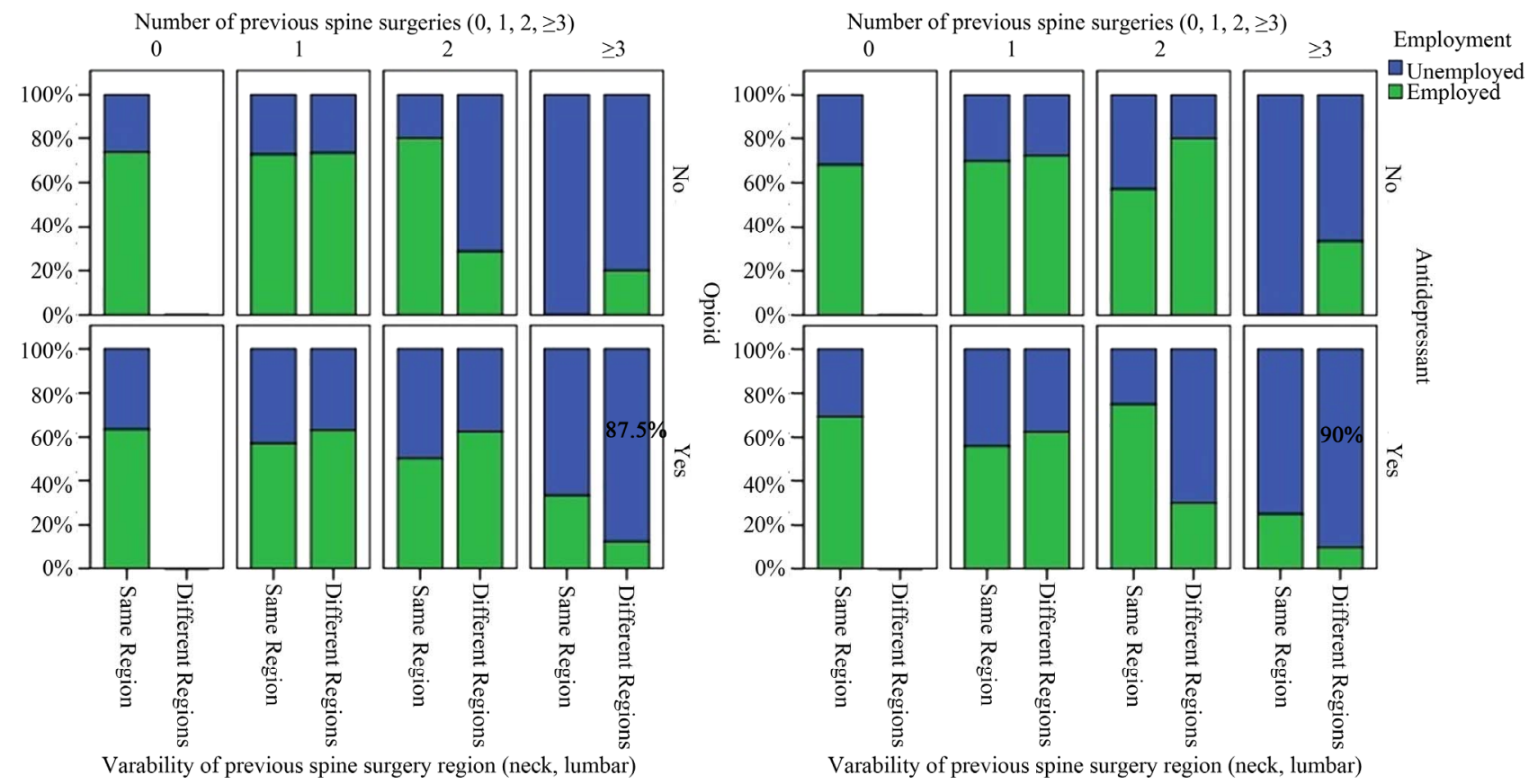

Figure 4. Increasing unemployment rate with increasing number of spine interventions and variability of intervention location in working age female patients on opioids and antidepressants.

characteristics, abuse severity, functioning profile and duration of treatment of men and women taking opioid [9] and antidepressant medications [10]. In this regard, a critical rectification in the methodology of outcome analysis studies appears mandatory by focusing on the possible role of different psychotropic drugs in connection with employment status of male and female spine surgery patients.

In light of the huge cost of spine surgery [11] and the indirect costs to society of absenteeism from work attributable to back problems [12], we are calling upon researchers to pay more attention to the negative relationship between employment status and the number of spine surgical interventions in their clinical investigations. We speculate that repeated surgical intervention on the spine of chronically complaining patients may accelerate the decline of their functional reserves resulting in quick disability and loss of employment capacity. This hypothesis needs to be verified in a randomized controlled prospective trial comparing the natural disease progress versus surgical treatment with inclusion of reoperation as independent marker and employment status as the outcome variable. Such a study may however not be feasible due to the practical and ethical difficulties inherent in it.

\section{REFERENCES}

[1] D. C. Cherkin, R. A. Deyo, J. D. Loeser, T. Bush and G. Waddell, "An International Comparison of Back Surgery Rates,” Spine, Vol. 19, No. 11, 1994, pp. 1201-1206.
[2] D. T. Gray, R. A. Deyo, W. Kreuter, S. K. Mirza, P. J. Heagerty, B. A. Comstock and L. Chan, "Population-Based Trends in Volumes and Rates of Ambulatory Lumbar Spine Surgery," Spine, Vol. 31, No. 17, 1964, pp. 1957-1963. doi:10.1097/01.brs.0000229148.63418.c1

[3] U.S. Department of Labor, Bureau of Labor Statistics. November 4, 2011.

[4] P. J. Watson, C. K. Booker, L. Moores and C. J. Main, "Returning the Chronically Unemployed with Low Back Pain to Employment," European Journal of Pain, Vol. 8, No. 4, 2004, pp. 359-369. doi:10.1016/j.ejpain.2003.11.003

[5] P. L. Sanderson, B. D. Todd, G. R. Holt and C. J. Getty, Compensation, Work Status, and Disability in Low Back Pain Patients," Spine, Vol. 20, No. 5, 1995, pp. 554-556. doi:10.1097/00007632-199503010-00009

[6] M. S. Walid and N. Zaytseva, "The Relationship of Unemployment and Depression with History of Spine Surgery," Perm Journal, Vol. 15, No. 1, 2011, pp. 19-22.

[7] M. S. Walid, J. S. Robinson, M. Abbara, A. Tolaymat and J. S. Jr. Robinson, "De Novo Spine Surgery as a Predictor of Additional Spine Surgery at the Same or Distant Spine Regions," German Medical Science, Vol. 9, 2011, p. 20.

[8] Disability Statistics. Council of Disability Awareness 2010.

http://www.disabilitycanhappen.org/chances_disability/di sability_stats.asp

[9] S. E. Back, R. L. Payne, A. H. Wahlquist, R. E. Carter, Z. Stroud, L. Haynes, M. Hillhouse, K. T. Brady and W. Ling, "Comparative Profiles of Men and Women with Opioid Dependence: Results from a National Multisite Effectiveness Trial," American Journal of Drug and Alcohol Abuse, Vol. 37, No. 5, 2011, pp. 313-323. 
doi:10.3109/00952990.2011.596982

[10] M. Tournier, A. Cougnard, S. Boutouaba-Combe and H. Verdoux, "Duration of Antidepressant Drug Treatment and Its Determinants in France," Encephale, Vol. 37, Suppl. 1, 2011, pp. S36- S41. doi:10.1016/j.encep.2010.06.007

[11] E. Stranges, C. A. Russo and B. Friedman, "Procedures with the Most Rapidly Increasing Hospital Costs, 2004-2007,"
Healthcare Cost and Utilization Project (HCUP) Statistical Briefs, Agency for Health Care Policy and Research, Rockville, 2006-2009.

[12] R. A. Deyo and Y. J. Tsui-Wu, "Descriptive Epidemiology of Low-Back Pain and Its Related Medical Care in the United States," Spine, Vol. 12, No. 9, 1987, pp. 264268. doi:10.1097/00007632-198704000-00013 